\title{
Vitexin attenuates acute doxorubicin cardiotoxicity in rats via the suppression of oxidative stress, inflammation and apoptosis and the activation of FOXO3a
}

\author{
ZHAN SUN ${ }^{1}$, BIN YAN $^{2}$, WEN YAN YU ${ }^{3}$, XUEPING YAO $^{1}$, XIAOJUAN MA $^{3}$, GELI SHENG $^{4}$ and QI MA ${ }^{1}$ \\ ${ }^{1}$ Laboratory of Functional Experimentation, Preclinical College, Xinjiang Medical University; ${ }^{2}$ Department of \\ Otorhinolaryngology, Ürümqi First People's Hospital; ${ }^{3}$ Department of Pathophysiology, Preclinical College, \\ Xinjiang Medical University, Ürümqi, Xinjiang $830054 ;{ }^{4}$ Department of Geriatrics, \\ Xinjiang National Clinical Research Base of Traditional Chinese Medicine, \\ Xinjiang Medical University, Ürümqi, Xinjiang 830000, P.R. China
}

Received April 21, 2015; Accepted June 23, 2016

DOI: $10.3892 / \mathrm{etm} .2016 .3518$

\begin{abstract}
Doxorubicin (DOX) is one of the most effective chemotherapeutic drugs. However, its clinical use has been hampered due to the development of cardiotoxicity. Vitexin, which is the active ingredient of hawthorn leaf extract, has various biological activities, including antioxidant and anti-inflammatory actions. The present study aimed to investigate whether vitexin was able to protect against DOX-induced acute cardiotoxicity in model rats and the mechanisms of this protective effect were assessed. Adult Sprague-Dawley rats were randomly assigned into the control (saline only), model (DOX only) and vitexin-treated (DOX plus vitexin) groups. Rats in the model and vitexin-treated groups were injected with DOX (2 mg/kg; i.p.) once a week for 4 weeks. Rats in the vitexin-treated group were administered $30 \mathrm{mg} / \mathrm{kg}$ oral vitexin once daily at doses for 4 weeks. The levels of lactate dehydrogenase, creatine kinase isoenzyme-MB, malondialdehyde, superoxide dismutase, catalase and myeloperoxidase were assessed using assay kits. The levels of inflammatory mediators, including tumor necrosis factor- $\alpha$, interleukin (IL)- $1 \beta$, IL-6, nuclear factor (NF)- $\mathrm{KB}$, and caspase-3, were assayed by the enzyme-linked immunosorbent assay method. Western blot analysis was performed to assess the protein expression levels of p-FOXO3a. Vitexin pretreatment significantly protected against DOX-induced myocardial damage, which was characterized by increased serum creatine kinase isoenzyme-MB and lactate dehydrogenase. Vitexin significantly ameliorated oxidative stress injury evoked by DOX, demonstrated by the
\end{abstract}

Correspondence to: Ms. Qi Ma, Laboratory of Functional Experimentation, Preclinical College, Xinjiang Medical University, 393 Xinyi Road, Ürümqi, Xinjiang 830054, P.R. China

E-mail: maqiqqq@163.com

Key words: doxorubicin, vitexin, cardiotoxicity, oxidative stress, inflammation, apoptosis inhibition of lipid peroxidation and the elevation of antioxidant enzyme activities. Furthermore, DOX provoked inflammatory responses by increasing the expression levels of IL-1 $\beta$, IL-6, NF- $\kappa \mathrm{B}$ and tumor necrosis factor- $\alpha$, whereas vitexin pretreatment significantly inhibited these inflammatory responses. Notably, DOX induced apoptotic tissue damage by increasing caspase-3 activity, whereas vitexin administration was able to decrease caspase-3 activity. In addition, vitexin induced elevated FOXO3a protein expression levels in the vitexin-treated group. In conclusion, the findings of the present study suggested that vitexin possesses cardioprotective action against DOX-induced cardiotoxicity by suppressing oxidative stress, inflammation and apoptotic signals.

\section{Introduction}

Doxorubicin (DOX), which belongs to the anthracyclines family, has been used to treat cancer since the late 1960s. It is a well-established and highly effective antineopalstic agent that is used to treat various adult and pediatric cancers, including solid tumors, leukemia, lymphomas and breast cancer (1). However, DOX causes various toxic effects, the most common of which is cardiotoxicity (2). Multiple mechanisms are involved in DOX-induced cardiomyopathy, including the increase in cardiac oxidative stress, as evidenced by reactive oxygen species that induce damage such as lipid peroxidation, and changes in adenylate cyclase activity leading to apoptosis and inflammation-related signaling pathways $(3,4)$. It has previously been suggested that DOX also elicits inflammatory effects by increasing the expression levels of nuclear factor kappa-B $(\mathrm{NF}-\mathrm{\kappa B})$, and induces the production of various proinflammatory mediators, including tumor necrosis factor (TNF)- $\alpha$ (5).

It has been demonstrated that DOX cardiotoxicity involves cardiomyocyte apoptosis (6). Caspase activity can be influenced by DOX, and caspase-3 activation is associated with DOX administration (7). The forkhead box (FOX) family of transcription factors regulate numerous cellular functions. These transcription factors are associated with the regulation 
of metabolism, cell proliferation, resistance of stress, immune system regulation, and apoptosis (8). FOXO3a is regulated by a number of signaling pathways, including extracellular signal-regulated kinase (ERK), Akt, IкB kinase, and serum glucocorticoid-related kinases $(9,10)$. Furthermore, FOXO3a is involved in resistance to oxidative stress and also linked to apoptotic processes by modulating the expression levels of proapoptotic and antiapoptotic proteins that regulate antioxidant enzyme levels, including mitochondrial antioxidant manganese superoxide dismutase (MnSOD) (11-13).

Hawthorn, of the rosaceae plant family, is a traditional Chinese medicine that is used to promote digestion (14). It has been reported that the ketone compounds extracted from the Hawthorn leaves are able to regulate blood lipids, blood pressure, increase coronary flow and protect the ischemic myocardium (15-17). Vitexin is the active ingredient extracted from hawthorn leaves, and it has previously been demonstrated that vitexin has a protective effect against hypoxia in the reoxygenation of myocardial cells (18). Therefore, the present study aimed to investigate the potential protective effect of vitexin against DOX-induced cardiotoxicity in rats and to elucidate the underlying molecular mechanisms in terms of oxidative stress, inflammatory and apoptotic mediators.

\section{Materials and methods}

Materials. Vitexin with a purity of $95 \%$ (Sigma-Aldrich, St. Louis, MO, USA) was dissolved in normal saline. The chemical structure of vitexin is presented in Fig. 1. Casein kinase (CK), lactate dehydrogenase (LDH), tumor necrosis factor- $\alpha$ (TNF- $\alpha$ ), interleukin (IL)-1 $\beta$, IL-6, nuclear factor kappa B (NF- $\mathrm{kB})$, malondialdehyde (MDA), SOD, catalase (CAT) and myeloperoxidase (MPO) commercial kits were purchased from Invitrogen (Thermo Fisher Scientific, Inc., Waltham, MA, USA).

Animals and modeling. A total of 36 male Sprague-Dawley rats (age, 6-8 weeks; weight, $260 \pm 20 \mathrm{~g}$ ) were provided by the Laboratory Animal Center of Xinjiang Medical University (Xinjiang, China). All animal care and experimental procedures were approved by the Animal Care Committee of Xinjiang Medical University (Ürümqi, China). Rats were maintained at $24 \pm 1^{\circ} \mathrm{C}$ (humidity, $40-80 \%$ ) under pathogen-free conditions with a $12 \mathrm{~h}$ light/dark cycle and ad libitum access to food and water. Rats were randomly and equally assigned into the control, model, and vitexin-treated groups. Control rats received an equal volume of normal saline by intraperitoneal (i.p.) injection at the same time points. Model group rats were induced by i.p. injection of DOX $(2 \mathrm{mg} / \mathrm{kg}$ ) once a week for 4 weeks. Vitexin-treated group rats were administered oral vitexin once daily at doses of $30 \mathrm{mg} / \mathrm{kg}$ for 4 weeks (19). Following treatment, blood samples were collected from the abdominal aorta and the rats were sacrificed via an overdose of ethyl carbamate prior to the harvesting of myocardial tissue. Blood samples were anticoagulated with EDTA and centrifuged at $3,000 \mathrm{x}$ g for $10 \mathrm{~min}$ at $4^{\circ} \mathrm{C}$, and the plasma was subsequently stored at $-80^{\circ} \mathrm{C}$ until further use.

Assessment of cardiotoxicity indices. LDH and creatine kinase isoenzyme-MB (CK-MB) levels were assessed in

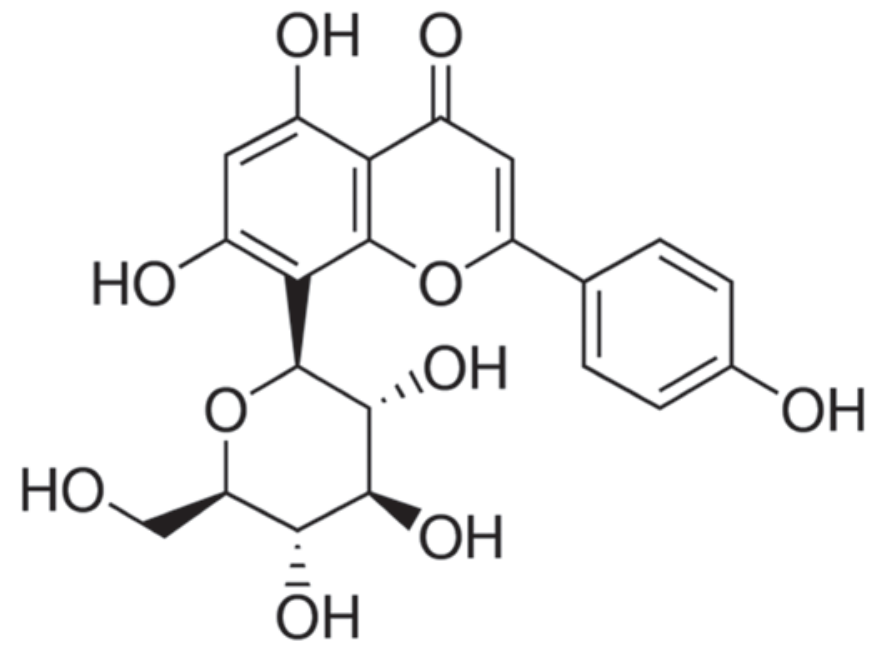

Figure 1. Chemical structure of vitexin.

serum samples using commercially available kits according to the manufacturer's protocol. All measurements were performed in duplicate.

Assessment of inflammatory cytokines in blood. TNF- $\alpha$, IL- $1 \beta$, IL-6 and NF- $\kappa$ B levels in the blood samples were determined using ELISA kits, according to the manufacturer's protocol. All measurements were performed in duplicate.

Assessment of oxidative stress markers and antioxidant enzyme activities. Lipid peroxidation was determined by estimating the level of thiobarbituric acid reactive substances measured as MDA, according to the manufacturer's protocol. Results were expressed as MDA (nmol)/mg of wet tissue. Cardiac SOD activity was determined according to the method outlined by Flohe and Otting (20). Values were expressed as U/mg protein. Cardiac CAT activity was assessed via the determination of the $\mathrm{H}_{2} \mathrm{O}_{2}$ decomposition rate at $240 \mathrm{~nm}$ and the values were expressed as U/mg protein. MPO activity was assayed using a commercially available kit, according to the manufacturer's protocol.

Assessment of cardioprotective FOXO3a protein expression levels by western blotting. Briefly, the cardiac left ventricular (LV) tissue was homogenized with a lysis buffer containing $25 \mathrm{mM}$ Tris, (pH 7.4), $150 \mathrm{mM} \mathrm{NaCl}, 5 \mathrm{mM}$ EDTA, $1 \mathrm{mM}$ $\mathrm{Na}_{3} \mathrm{VO}_{4}, 10 \mathrm{mM} \mathrm{NaF}, 1 \%$ (vol/vol) Triton X-100, and $1 \%$ (vol/vol) glycerol. Equal amounts of the heart homogenate $(30 \mu \mathrm{g})$ were separated by $10 \%$ SDS-PAGE (wt/vol) and subsequently transferred onto a nitrocellulose membrane (Trans-Blot Transfer Medium; Bio-Rad Laboratories, Inc., Hercules, CA, USA), and blocked with 5\% skimmed milk at room temperature for $60 \mathrm{~min}$. Membranes were washed three times for 5 min with Tris-buffered saline with Tween 20 (TBS-T) and incubated overnight with the appropriate primary anti-phosphorylation-FOXO3a (p-FOXO3a; $1: 2,000 ; 9464)$ and anti- $\beta$-actin (1:500; 8457; both American Diagnostica Inc., Stamford, CT, USA) antibodies at $4^{\circ} \mathrm{C}$. Subsequently, the membranes were washed thrice with TBS-T and incubated with secondary antibodies for $2 \mathrm{~h}$ at room temperature. Immunodetection was performed using horseradish peroxidase-conjugated secondary 
A

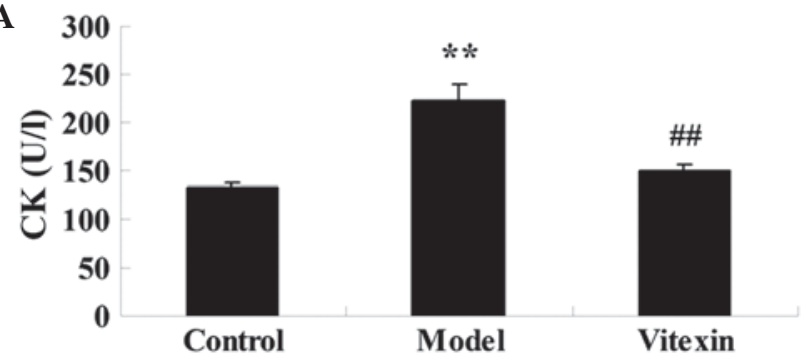

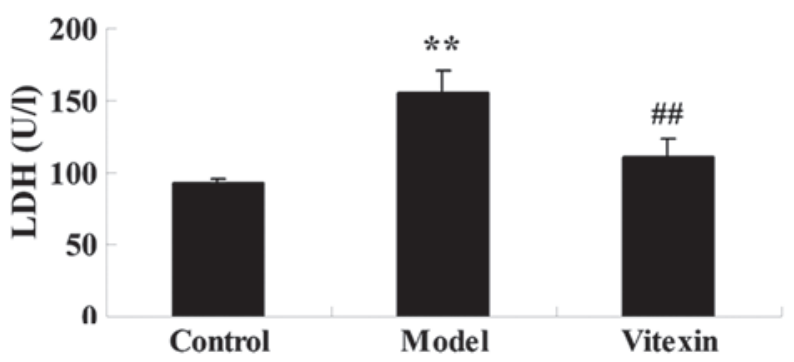

Figure 2. Cardiotoxicity markers, (A) casein kinase (CK) and (B) lactate dehydrogenase (LDH), for rats treated with doxorubicin and $/ \mathrm{or} 30 \mathrm{mg} / \mathrm{kg}$ vitexin. ${ }^{* *} \mathrm{P}<0.01$ vs. the control group; ${ }^{\# /} \mathrm{P}<0.01$ vs. the model group.

A

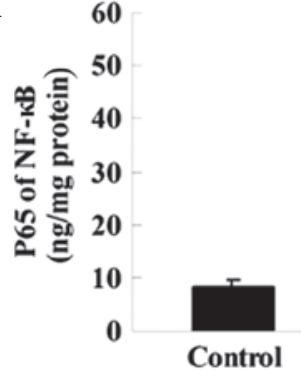

C

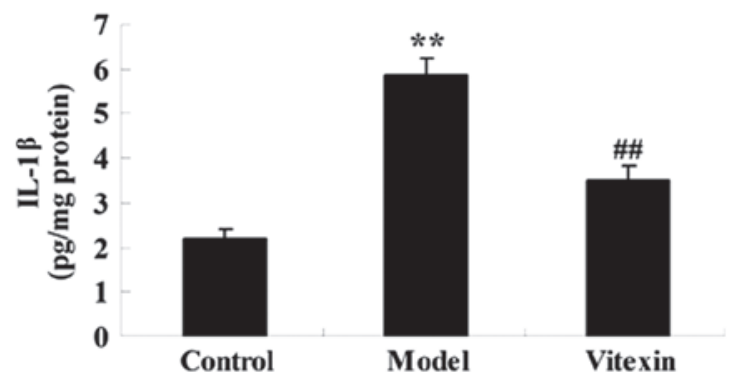

B

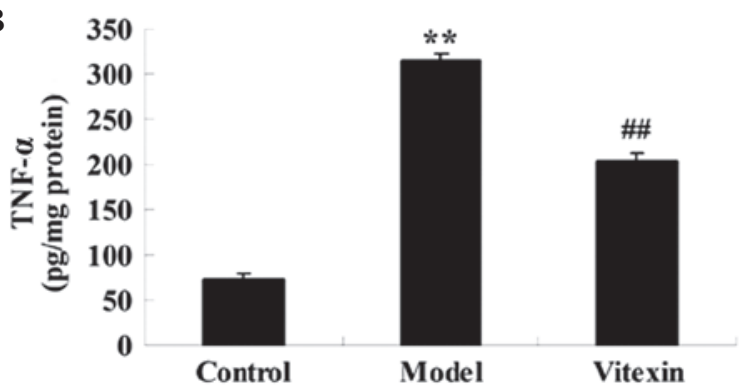

D

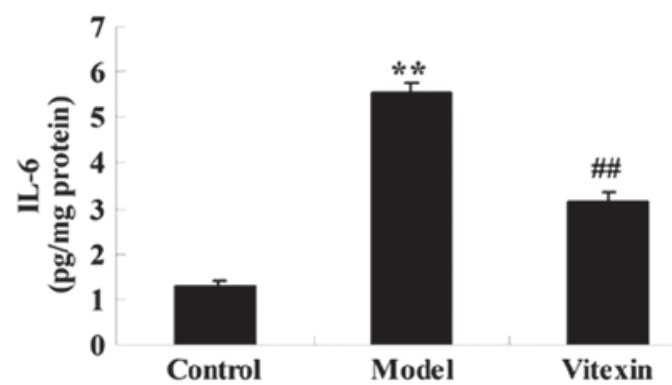

Figure 3. Effect of $30 \mathrm{mg} / \mathrm{kg}$ vitexin on inflammatory cytokines in rats, including (A) NF- $\mathrm{kB}$, (B) TNF- $\alpha$, (C) IL-1 $\beta$ and (D) IL-6. ${ }^{* *} \mathrm{P}<0.01 \mathrm{vs.} \mathrm{the} \mathrm{control}$ group; ${ }^{\# \#} \mathrm{P}<0.01$ vs. the model group.

antibody (1:2,000; 5522; Cell Signaling Technology Inc., Danvers, MA, USA) using an enhanced chemiluminescence kit (GE Healthcare Life Sciences, Chalfont, UK). Blot quantification was performed using ImageQuant LAS 500 software (GE Healthcare Life Sciences).

Assessment of apoptotic markers. ELISA kits were used to analyze the levels of caspase-3 in myocardial tissue. Briefly, cardiac LV tissue was homogenized with a lysis buffer and equal amounts of the heart homogenate $(30 \mu \mathrm{g})$ were supplemented with reaction buffer with 1 Asp-Glu-Val-Asp (DEVD)-p-nitroaniline and incubated at $37^{\circ} \mathrm{C}$ for $6 \mathrm{~h}$. Caspase-3 activation was measured using a microplate reader (Bio-Rad Laboratories, Inc.) at an absorbance of $405 \mathrm{~nm}$.

Statistical analysis. Results are presented as the mean \pm standard deviation. For tests of significance between the groups, one-way analysis of variance was performed. Comparisons between two groups were performed using unpaired Student's t-test. SPSS 17.0 statistical software (SPSS, Inc., Chicago, IL, USA) was used to conduct statistical analyses. $\mathrm{P}<0.05$ was considered to indicate a statistically significant difference. All measurements were performed at least three independent times.

\section{Results}

Biochemical cardiotoxicity markers. Serum markers indicating myocardial injury, LDH and CK-MB activities were assessed. As shown in Fig. 2, the activities of LDH and CK-MB were significantly increased in the serum of the DOX group, as compared with the control group. Pretreatment with vitexin resulted in a significant reduction in serum levels, as compared with the DOX group. Rats in the vitexin group did not exhibit any significant changes in LDH and CK-MB levels, as compared with the control group.

Effect of vitexin on inflammatory cytokines. The concentration levels of TNF- $\alpha$, IL-1 $\beta$, IL- 6 and NF- $\kappa B$ in the blood represent proinflammatory mediators, which are thought to have important roles in the development of ischemic heart failure (21). As shown in Fig. 3, TNF- $\alpha$, IL-1 $\beta$, IL-6 and NF-кB levels markedly increased in the model group, as compared with the control group, whereas these levels were decreased in the vitexin group.

Oxidative stress markers and antioxidant enzymes. As shown in Fig. 4A, MDA levels significantly increased in the model group, as compared with the control group $(\mathrm{P}<0.01)$, and vitexin 
A

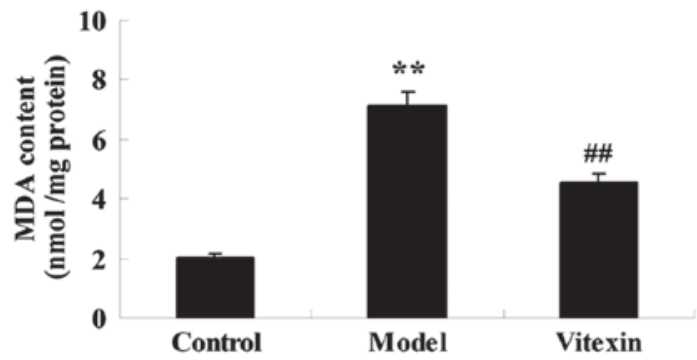

C

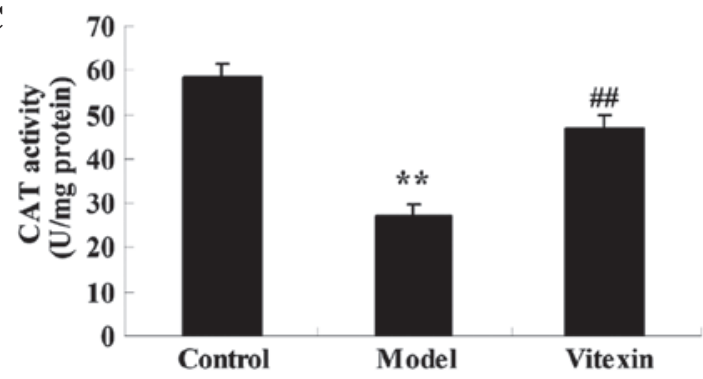

B

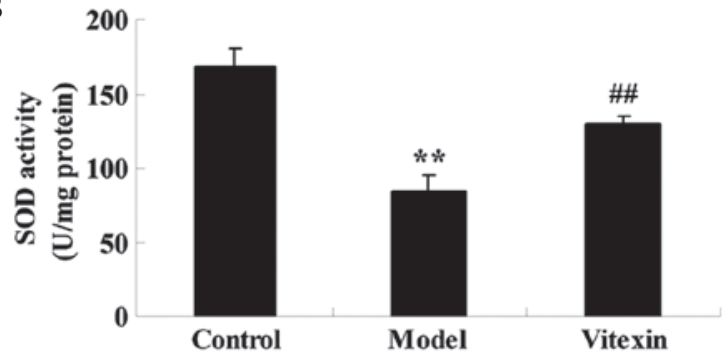

D

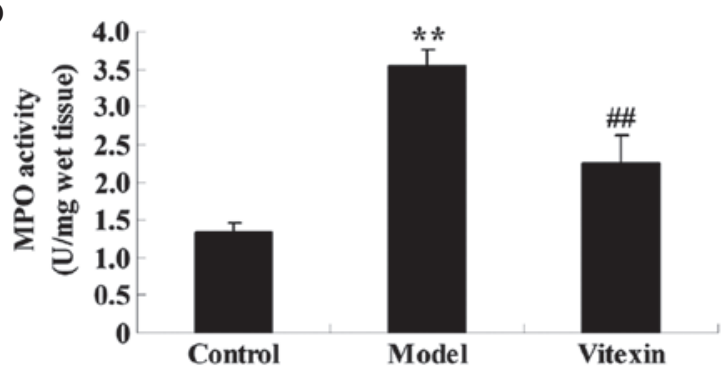

Figure 4. Oxidative stress markers in rats treated with doxorubicin and/or $30 \mathrm{mg} / \mathrm{kg}$ vitexin, including (A) malondialdehyde (MDA), (B) superoxide dismutase (SOD), (C) catalase (CAT) and (D) myeloperoxidase (MPO) in rats. ${ }^{* *} \mathrm{P}<0.01$ vs. the control group; ${ }^{\# \# P<0.01 ~ v s . ~ t h e ~ m o d e l ~ g r o u p . ~}$

A

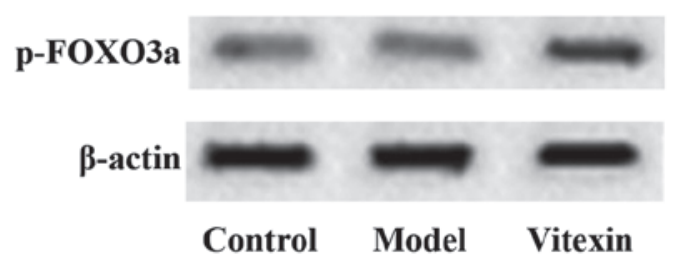

B

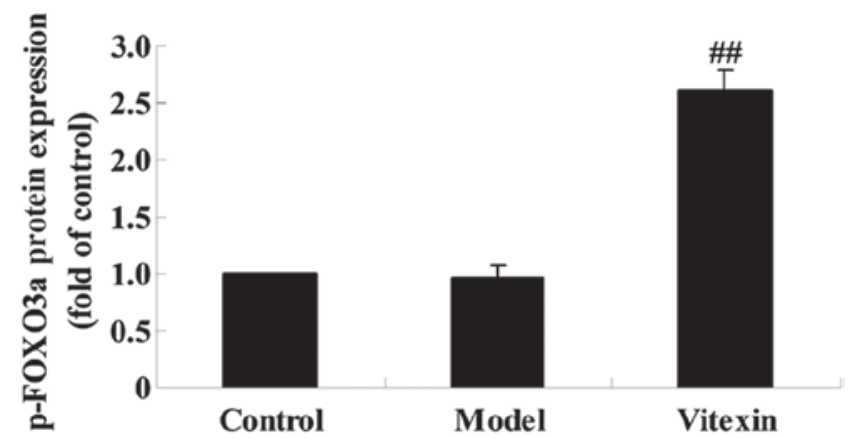

Figure 5. Effect of vitexin on FOXO3a in rats. Effect of $30 \mathrm{mg} / \mathrm{kg}$ vitexin on the protein expression levels of FOXO3a was assessed by (A) western blotting and (B) subsequent quantitative analysis in a rat model of ischemic heart failure. ${ }^{\# \#} \mathrm{P}<0.01$ vs. the model group.

significantly inhibited MDA levels as compared with the model group $(\mathrm{P}<0.01)$. As shown in Fig. $4 \mathrm{~B}$ and $\mathrm{C}$, assessment of the myocardial antioxidant enzymatic profile of rats in the DOX group demonstrated a significant reduction in CAT and SOD activities, as compared with the control group $(\mathrm{P}<0.01)$. Pretreatment with vitexin significantly restored CAT and SOD activities, as compared with the DOX group $(\mathrm{P}<0.01)$. As shown in Fig. 4D, MPO activity significantly increased in the model group, as compared with the control group $(\mathrm{P}<0.05)$; however, MPO activity was significantly deceased following vitexin treatment. These results indicated that vitexin may protect heart function by inhibiting oxidative stress.

Effect of vitexin on FOXO3a. To investigate whether vitexin is able to modulate the expression of p-FOXO3a protein, western blot analysis was performed. The intensity measurement for proteins was determined according to the ratio of the integrated intensity of the p-FOXO3a band to the integrated intensity of the $\beta$-actin band in the same sample. As shown in Fig. 5A and B, there was no significant difference between the

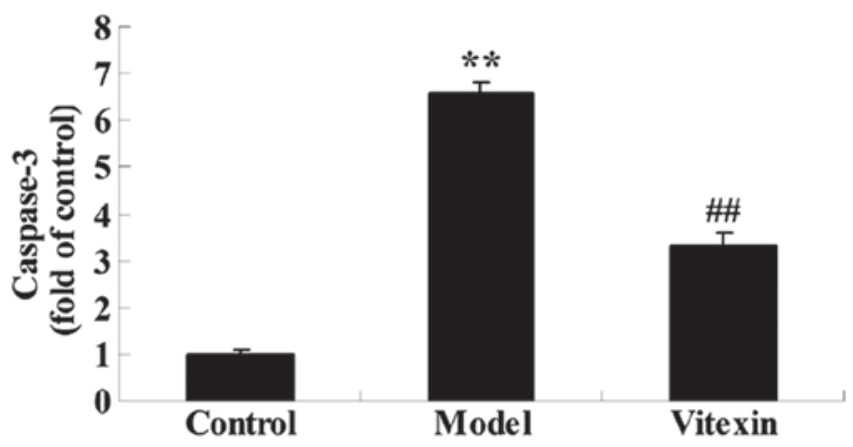

Figure 6. Effect of $30 \mathrm{mg} / \mathrm{kg}$ vitexin on caspase-3 levels in rats. ${ }^{* *} \mathrm{P}<0.01 \mathrm{vs}$. the control group; ${ }^{\# \#} \mathrm{P}<0.01$ vs. the model group.

model and control groups; however, the expression levels of $\mathrm{p}-\mathrm{FOXO} 3 \mathrm{a}$ in the vitexin group were significantly increased compared with the control and model groups $(\mathrm{P}<0.01)$. These results indicated that the protective effect induced by vitexin against DOX-induced cardiac failure may be associated with p-FOXO3a protein expression. 
Apoptotic markers. As shown in Fig .6, caspase-3 activation significantly increased in the model group, as compared with the control group $(\mathrm{P}<0.01)$; however, these increased caspase-3 levels were significantly reduced by pretreatment with vitexin, as compared with the DOX model group $(\mathrm{P}<0.01)$. This result indicated that the protective effect induced by vitexin against DOX-induced cardiac failure may be associated with an anti-apoptotic mechanism.

\section{Discussion}

Doxorubicin (DOX) is an effective chemotherapeutic agent that is frequently used to treat various malignancies. However, its clinical use is hampered by the development of cardiotoxicity. It has previously been demonstrated that DOX-induced cardiotoxicity occurs through mechanisms other than those that mediate its antitumor effect (22). The present study aimed to investigate the potential cardioprotective effect of vitexin against DOX-induced cardiotoxicity in rats and the underlying mechanisms. The present findings indicated that pretreatment with vitexin prior to treatment with DOX for 4 weeks improved the cardiac function of rats, which included a decrease of LDH and CK-MB in serum. Notably, the preservation of heart function was demonstrated to be associated with a decrease in oxidative stress and the apoptosis in cardiomyocytes as well as a decrease in inflammatory cytokines levels.

DOX-induced heart failure is characterized by the generation of free radicals in the cardiac tissue $(22,23)$. The present data demonstrated that the activity of SOD and CAT were significantly decreased in the DOX group and the co-treatment of vitexin increased SOD and CAT activity. MDA is a lipid peroxidation marker that is used to assess lipid peroxidation due to increased oxidative stress (24). In the present study, blood levels of MDA were markedly increased in the DOX group, and this was significantly reversed by pretreatment with vitexin. MPO is an enzyme that is predominantly located in the primary granules of neutrophils and its main function is to kill microorganisms; however, under certain conditions, it produces excess oxidant, which leads tissue damage (25). In the present study, MPO activity significantly increased after DOX administration. In contrast, pretreatment with vitexin significantly decreased MPO activity and reduced neutrophil infiltration. These findings suggested that vitexin is a potential antioxidant molecule that may be used to protect the heart from DOX-induced failure.

Oxidative stress can trigger inflammatory cascades, which are primarily mediated via NF- $\kappa \mathrm{B}(26,27)$. NF- $\kappa \mathrm{B}$ is a key transcription factor that regulates inflammatory processes (28). Various studies have reported that $N F-\kappa B$ is involved in the pathogenesis of heart failure $(29,30)$. Activation of $N F-\kappa B$ induces the activation of genetic programs that lead to the transactivation of cytokines and chemokines. The present data demonstrated that inflammatory cytokines levels increased in the DOX group, as compared with the control group; whereas these levels decreased in the vitexin group, as compared with the DOX group.

Oxidative stress evoked by DOX results in the apoptotic death of cardiomyocytes $(31,32)$ through various signaling pathways, including the activation of caspase-3 (33). The present findings demonstrated that caspase- 3 activation increased in the model group, as compared with the control group; however, these increased caspase-3 levels were significantly reduced by pretreatment with vitexin, as compared with the DOX group. These findings indicated that vitexin pretreatment may protect the heart by decreasing the apoptotic rate of cardiomyocytes in response to DOX.

FOXO3a has an important role in the mechanisms which protect cells from oxidative stress-induced cell death. FOXO3a is regulated by a number of signaling pathways, including ERK, Akt, I $\kappa \mathrm{B}$ kinase, and serum glucocorticoid-related kinases (7). FOXO3a regulates the levels of antioxidant enzymes, including MnSOD (34). The results of the present study demonstrated that there were no significant differences between the model and control groups; however, the expression levels of p-FOXO3a in the vitexin group increased, as compared with the control and model groups. These results indicate that the protection against DOX-induced cardiotoxicity may be associated with p-FOXO3a protein expression.

In conclusion, these results demonstrated that vitexin may be an effective therapeutic agent against DOX-induced cardiotoxicity. The mechanisms investigated included the attenuation of oxidative stress, reducing cardiac inflammatory cytokines, increased FOXO3a, and inhibition of caspase-3 activation. We propose that vitexin may be used as an effective therapeutic agent to prevent DOX-induced cardiomyopathy.

\section{Acknowledgements}

The present study was supported by the National Natural Science Foundation of China (grant no. 81560078), China Postdoctoral Science Foundation (grant no. 2013M532102), and Scientific Research Foundation for Doctors at Xinjiang Medical University (grant no. 201006)

\section{References}

1. Poprach A, Petrakova K, Vyskoýil J, Lakomý R, Nċmeýek R, Kocak I, Kocakova I and Vyzula R: Cardiotoxicity of drugs used in oncology. Klinicka Onkologie: Casopis Ceske a Slovenske Onkologicke Spolecnosti 21: 288-293, 2008 (In Czech).

2. Jain D: Cardiotoxicity of doxorubicin and other anthracycline derivatives. J Nucl Cardiol 7: 53-62, 2000.

3. Khan MA, Singh M, Khan MS, Ahmad W, Najmi AK and Ahmad S: Alternative approach for mitigation of doxorubicin-induced cardiotoxicity using herbal agents. Curr Clin Pharmacol 9: 288-297, 2014.

4. Nagai K, Fukuno S, Oda A and Konishi H: Protective effects of taurine on doxorubicin-induced acute hepatotoxicity through suppression of oxidative stress and apoptotic responses. Anticancer Drugs 27: 17-23, 2015.

5. Wang S, Kotamraju S, Konorev E, Kalivendi S, Joseph J and Kalyanaraman B: Activation of nuclear factor-kappaB during doxorubicin-induced apoptosis in endothelial cells and myocytes is pro-apoptotic: The role of hydrogen peroxide. Biochem J 367: 729-740, 2002.

6. Kalyanaraman B, Joseph J, Kalivendi S, Wang S, Konorev E and Kotamraju S: Doxorubicin-induced apoptosis: Implications in cardiotoxicity. Mol Cell Biochem 234-235: 119-124, 2002.

7. Ueno M, Kakinuma Y, Yuhki K, Murakoshi N, Iemitsu M, Miyauchi T and Yamaguchi I: Doxorubicin induces apoptosis by activation of caspase- 3 in cultured cardiomyocytes in vitro and rat cardiac ventricles in vivo. J Pharmacol Sci 101: 151-158, 2006.

8. van der Horst A and Burgering BM: Stressing the role of FoxO proteins in lifespan and disease. Nat Rev Mol Cell Biol 8: 440-450, 2007. 
9. Yang JY, Zong CS, Xia W, Yamaguchi H, Ding Q, Xie X, Lang JY, Lai CC, Chang CJ, Huang WC, et al: ERK promotes tumorigenesis by inhibiting FOXO3a via MDM2-mediated degradation. Nat Cell Biol 10: 138-148, 2008.

10. Yang W, Dolloff NG and El-Deiry WS: ERK and MDM2 prey on FOXO3a. Nat Cell Biol 10: 125-126, 2008.

11. Huang $\mathrm{H}$ and Tindall DJ: Dynamic FoxO transcription factors. J Cell Sci 120: 2479-2487, 2007.

12. Kops GJ, Dansen TB, Polderman PE, Saarloos I, Wirtz KW, Coffer PJ, Huang TT, Bos JL, Medema RH and Burgering BM: Forkhead transcription factor FOXO3a protects quiescent cells from oxidative stress. Nature 419: 316-321, 2002.

13. Marinkovic D, Zhang X, Yalcin S, Luciano JP, Brugnara C, Huber T and Ghaffari S: Foxo3 is required for the regulation of oxidative stress in erythropoiesis. J Clin Invest 117: 2133-2144, 2007.

14. Zick SM, Vautaw BM, Gillespie B and Aaronson KD: Hawthorn Extract Randomized Blinded Chronic Heart Failure (HERB CHF) trial. Eur J Heart Fail 11: 990-999, 2009.

15. Wang XS, Hu XC, Chen GL, Yuan X, Yang RN, Liang S, Ren J, Sun JC, Kong GQ, Gao SG, Feng XS: Effects of vitexin on the pharmacokinetics and mRNA expression of CYP isozymes in rats. Phytother Res 29: 366-372, 2014.

16. Je HG, Hong SM, Je HD, Choi YS, Seo SY, Min YS, Chung SJ, Shin YK, Lee TJ, Park ES and Jeong JH: The inhibitory effect of vitexin on the agonist-induced regulation of vascular contractility. Pharmazie 69: 224-228, 2014.

17. Tassell MC, Kingston R, Gilroy D, Lehane M and Furey A: Hawthorn (Crataegus spp.) in the treatment of cardiovascular disease. Pharmacogn Rev 4: 32-41, 2010.

18. Xue HF, Ying ZM, Zhang WJ, Meng YH, Ying XX and Kang TG: Hepatic, gastric, and intestinal first-pass effects of vitexin in rats. Pharm Biol 52: 967-971, 2014

19. Lu CC, Xu YQ, Wu JC, Hang PZ, Wang Y, Wang C, Wu JW, Qi JC, Zhang Y and Du ZM: Vitexin protects against cardiac hypertrophy via inhibiting calcineurin and CaMKII signaling pathways. Naunyn-Schmiedeberg Arch Pharmacol 386: 747-755, 2013.

20. Flohe L and Otting F: Superoxide dismutase assays. Methods Enzymol 105: 93-104, 1984.

21. Sharma HS and Das DK: Role of cytokines in myocardial ischemia and reperfusion. Mediators Inflamm 6: 175-183, 1997.

22. El-Sayed EM, Mansour AM and Abdul-Hameed MS: Thymol and carvacrol prevent doxorubicin-induced cardiotoxicity by abrogation of oxidative stress, inflammation, and apoptosis in rats. J Biochem Mol Toxicol 30: 37-44, 2016.
23. Gao Y, Xu Y, Hua S, Zhou S and Wang K: ALDH2 attenuates Dox-induced cardiotoxicity by inhibiting cardiac apoptosis and oxidative stress. Int J Clin Exp Med 8: 6794-6803, 2015.

24. Torun AN, Kulaksizoglu S, Kulaksizoglu M, Pamuk BO, Isbilen E and Tutuncu NB: Serum total antioxidant status and lipid peroxidation marker malondialdehyde levels in overt and subclinical hypothyroidism. Clin Endocrinol (Oxf) 70: 469-474, 2009.

25. Ma Z, Ji W, Fu Q and Ma S: Formononetin inhibited the inflammation of LPS-induced acute lung injury in mice associated with induction of PPAR gamma expression. Inflammation 36: 1560-1566, 2013

26. Munoz A and Costa M: Nutritionally mediated oxidative stress and inflammation. Oxid Med Cell Longev 610950, 2013.

27. Reuter S, Gupta SC, Chaturvedi MM and Aggarwal BB: Oxidative stress, inflammation, and cancer: How are they linked? Free Radic Biol Med 49: 1603-1616, 2010.

28. Barnes PJ and Karin M: Nuclear factor-kappaB: A pivotal transcription factor in chronic inflammatory diseases. N Engl J Med 336: 1066-1071, 1997.

29. Frantz S, Fraccarollo D, Wagner H, Behr TM, Jung P, Angermann CE, Ertl G and Bauersachs J: Sustained activation of nuclear factor kappa $B$ and activator protein 1 in chronic heart failure. Cardiovascular Res 57: 749-756, 2003.

30. Van der Heiden K, Cuhlmann S, Luong le A, Zakkar M and Evans PC: Role of nuclear factor kappaB in cardiovascular health and disease. Clinical Sci 118: 593-605, 2010.

31. Chen CT, Wang ZH, Hsu CC, Lin $\mathrm{HH}$ and Chen JH: In vivo protective effects of diosgenin against doxorubicin-induced cardiotoxicity. Nutrients 7: 4938-4954, 2015.

32. Gulimire A, Ybadaiti T, Rena K and Ting FW: Protective effect of total flavonoids of $H$. rhamnoides L. sunsp. Turkestanica rousi against adriamycin-induced cardiotoxicity in rats. Xin Jiang Yi Ke Da Xue Xue Bao 33: 383-385, 2010.

33. Dash SK, Chattopadhyay S, Ghosh T, Dash SS, Tripathy S, Das B, Bag BG, Das D and Roy S: Self-assembled betulinic acid protects doxorubicin induced apoptosis followed by reduction of ROS-TNF-alpha-caspase-3 activity. Biomed Pharmacother 72: 144-157, 2015.

34. Kim AD, Kang KA, Piao MJ, Kim KC, Zheng J, Yao CW, Cha JW, Hyun CL, Kang HK, Lee NH and Hyun JW: Cytoprotective effect of eckol against oxidative stress-induced mitochondrial dysfunction: involvement of the FoxO3a/AMPK pathway. J Cell Biochem 115: 1403-1411, 2014. 